\title{
Analysis of Joint and Hand Impedance During Teleoperation and Free-Hand Task Execution
}

\author{
Jacopo Buzzi, Cecilia Gatti, Giancarlo Ferrigno, Elena De Momi
}

\begin{abstract}
Teleoperated robotic surgery allows filtering and scaling the hand motion to achieve high precision during the surgical interventions. Teleoperation represents a very complex sensory-motor task, mainly due to the kinematic and kinetic redundancies that characterize the human motor control. It requires an intensive training phase to acquire sufficient familiarity with the master-slave architecture.

We estimated the hand stiffness modulation during the execution of a simulated suturing task in teleoperation, with two different master devices, and in free-hand. Kinematic data of eight right-handed users were acquired, using electromagnetic and optical tracking systems, and analysed using a musculoskeletal model. Through inverse dynamics, muscular activation was computed and used to obtain the joint torque and stiffness, leading to end-point stiffness estimation. The maximal stiffness value and its angular displacement with respect to the trajectory tangent was computed. The results show that there is a difference in how the main stiffness axis was modulated by using the two master devices with respect to free-hand, with higher values and variability for the serial link manipulator. Moreover, a directional modulation of the hand stiffness through the trajectory was found, showing that the users were aligning the direction of the main stiffness axis perpendicularly to the trajectory.
\end{abstract}

\section{INTRODUCTION}

Robot-Assisted Surgery (RAS) is achieving great outcomes and feedbacks [1]. Thanks to its advantages, such as augmented precision due to movement and force scaling, hand tremor and chopstick effect compensation [2], this technology has widely spread in the last decade. During teleoperated robotic surgery, the user interacts with a master controller that detects the position and motion of his/her hand and sends it to the slave robot. In comparison with traditional laparoscopic surgery, in which the tools usually have rigid shafts constrained to approach the surgery area from the direction of the incision, thus limiting the surgery tool mobility, the mechanical characteristics of the tools developed for robotic surgery allows for the reproduction of the human wrist mobility. These mechanical differences are reflected in substantial kinematic and motor control differences that lead to limited correlation between laparoscopic expertise and robotic surgery skills [3].

To be able to fully exploit the potential of this technique [4] and take advantage of the tools high dexterity (the capability of changing the position and orientation of the manipulated objects to every arbitrary chosen pose), surgeons have to undergo a long and intensive training phase [5]. During this process, teleoperators have to familiarise with the

The authors are with the Department of Electronics, Informatics and Bioengineering, Politecnico di Milano, Piazza Leonardo da Vinci, 20133 Milan, Italy jacopo.buzzi@polimi.it new environment, by means of, for example, compensating for the absence of haptic feedback [6] or learning how to fully exploit the 3D visualization. Researchers are trying to restore the lack of force feedback from the tool tip to the surgeon hand using different strategies [7], [8], involving kinaesthetic feedback [9], generating guiding forces [10] and actively changing the manipulator characteristics to help the surgeons [11], [12]. While these studies will help in narrowing the gap that exist between free-hand and teleoperated surgery [13], a better understanding of the kinematic and kinetic strategies that define the human interaction with the master controller could potentially suggest new solutions to improve the surgeon's experience.

Teleoperation is a challenging task for the human motor control system, encompassing the management of the intrinsic redundancy of the neuro-muscular-skeletal system. In fact, from a kinematic standpoint, the human arm can be modelled as a seven degrees of freedom (DoF) redundant manipulator, which allows to theoretically assume infinite joint configurations to achieve the same hand position and orientation. Moreover, from a kinetic standpoint, a large set of muscular activations produce the same motion, but involving different levels of arm stiffness. The different ways the central nervous system manages these redundancies are decided by human motor control strategies.

A promising approach in the study of the teleoperators' kinematic redundancy has been recently presented by Nisky et al. [14], [15]. The authors studied the arm joint configuration in repeated reaching tasks performed with the Da Vinci Si surgical system (Intuitive Surgical, Mountain View, California) and in free-hand, comparing not only the task precision performance in terms of end-effector control, but also the arm configuration variability. Through the Uncontrolled Manifold (UCM) analysis the authors were able to distinguish between two joint variability components: one that helped the expert users in hand stabilization and another that captured residual noise. Using the UCM analysis, the authors were able to weight the users' joint variability into task relevant and irrelevant manifolds, showing that experienced surgeons were able to maximise the ratio between task-irrelevant and task-relevant variability.

The central nervous system, while handling kinetic redundancy, is able to control the mechanical impedance of the arm also through mechanisms as muscle co-contraction. Teleoperation is usually characterized by slow and precise movements that can be divided in subsequent joint configurations, each characterized by a mechanical impedance dominated by stiffness. 
Several studies measured the arm stiffness resulting from small hand displacement in stationary and dynamic conditions [16],[17], underlying the body capabilities of restoring a desired configuration after being subjected to external perturbation. Stiffness is a measure of arm stability against noise and perturbation and its modulation has been studied during the execution of multijoint tasks requiring accuracy [18][19]. Other researches [20] also found that the arm stiffness decreases during multiple executions of the same tasks as far as the users learn. These results suggest that stiffness and, more in general, impedance control is a key factor in the process of learning and mastering the execution of a specific task.

Previous studies obtained the arm end-point stiffness applying known displacements to the subjects hand using robotic handles and acquiring the force exchanged by the hand with the robotic handle [21] [22]. Others evaluated the arm stiffness by analysing surface EMG activity of antagonist muscles [23]. In our study we propose a comparison between the arm end-point stiffness computed using musculoskeletal models during the execution of a 3D task with different master devices in teleoperation and in free-hand. Using freehand as a reference of the most natural and transparent controlling strategy, stiffness values and principal direction were compared and correlated with the task execution performance.

\section{MATERIALS AND METHODS}

\section{A. Task}

The task was designed with the aim of recreating the wrist movement performed during suturing, where the needle is inserted through the tissues with a wrist rotation. Users were asked to follow an U-shaped trajectory with a ringterminating tool and to sweep the ring on the path while keeping the ring centre as more aligned as possible with the trajectory. The shape and length of the path were grossly estimated reaching a compromise between wrist activation and task feasibility.

\section{1) The Virtual Task}

Fig. 1(a) shows the virtual trajectory: the starting point, in the rightmost position of the trajectory, is markerd with a green sphere, the user had to reach the red sphere on the left. During the task execution, users were given visual feedback which consisted in a change of the trajectory color based on the distance between the center of the tool ring and the trajectory: from light green, when the trajectory is in the middle of the ring, to red, when a collision was detected.

The virtual task was developed using V-Rep (Coppelia Robotics GmbH, Zrich, Switzerland), a robot simulation program that can be interfaced through the Robotic Operating System (ROS) [24] network with the master devices involved in the test and with the data acquisition framework.

\section{2) The free-hand Task}

In order to recreate the same task for the free-hand movements, the trajectory was realised using a wooden base and coated metal wires that were shaped to assume the same

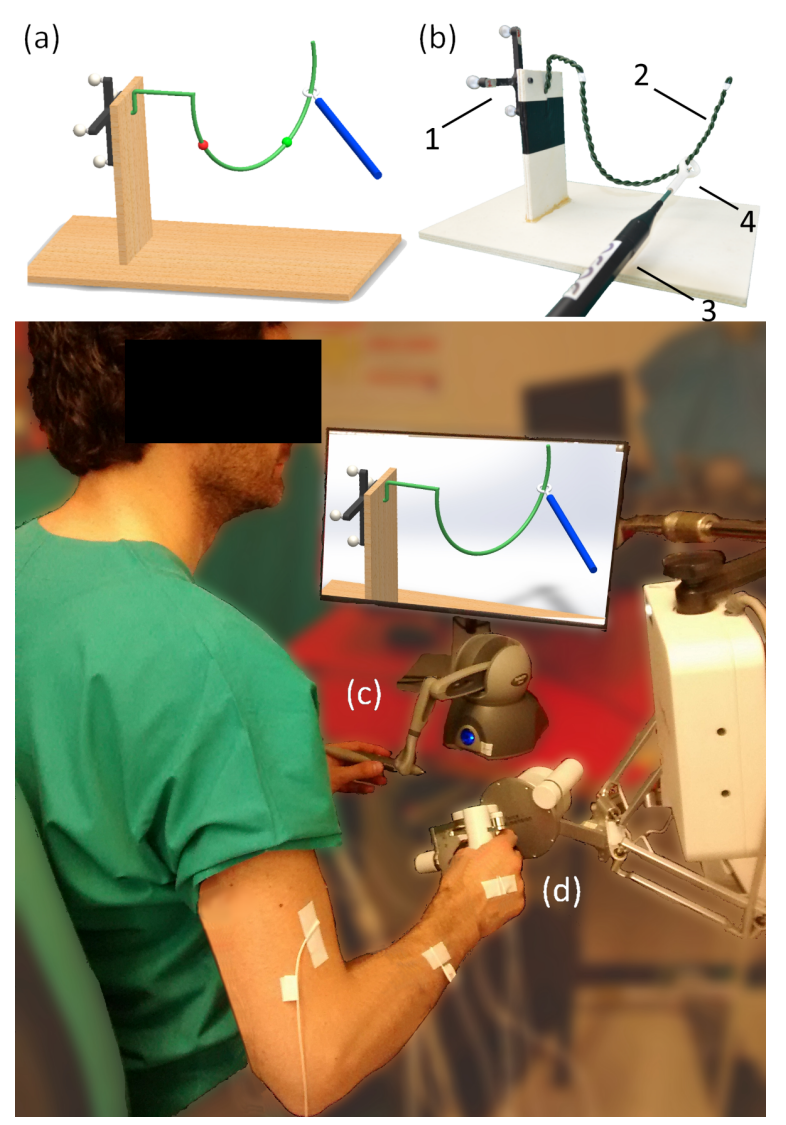

Fig. 1. The complete setup: (a)The virtual reality trajectory. (b) The freehand task: 1. Base 2. Metal wire trajectory 3. EMT Pointer 4. 3D Printed ring end-effector (c) The serial link master device (SL). (d) The parallel link master device (PL).

dimensions and shape of the virtual one. The user performed the task using a tracked fast prototyped tool with the same dimensions as the virtual one (see Fig. 1(b)).

\section{B. Experimental protocol}

Eight users ( 3 males, 5 females, $24 \pm 1.3$ year old) were recruited during the experimental campaign; all of them were right-handed novices with no surgical experience and no previous experience with haptic interfaces or teleoperation. This study was carried out in accordance with the recommendations of our institution with written informed consent from the subjects in accordance with the declaration of Helsinki. Each user performed 10 repetitions of the task with three control modalities:

1) Parallel Link robot (PL)

2) Serial Link robot (SL)

3) Free-hand (FH)

The users were given information about how to control the device and about the visual and haptic feedback that they were going to receive. In PL and SL the users had 5 to 15 minutes to gain a sufficient acquaintance with the control and with the virtual environment. During FH, the initial training time was limited to about 1 minute.

In PL, SL and FH, the users were instructed to find a compromise between task execution time and accuracy. 


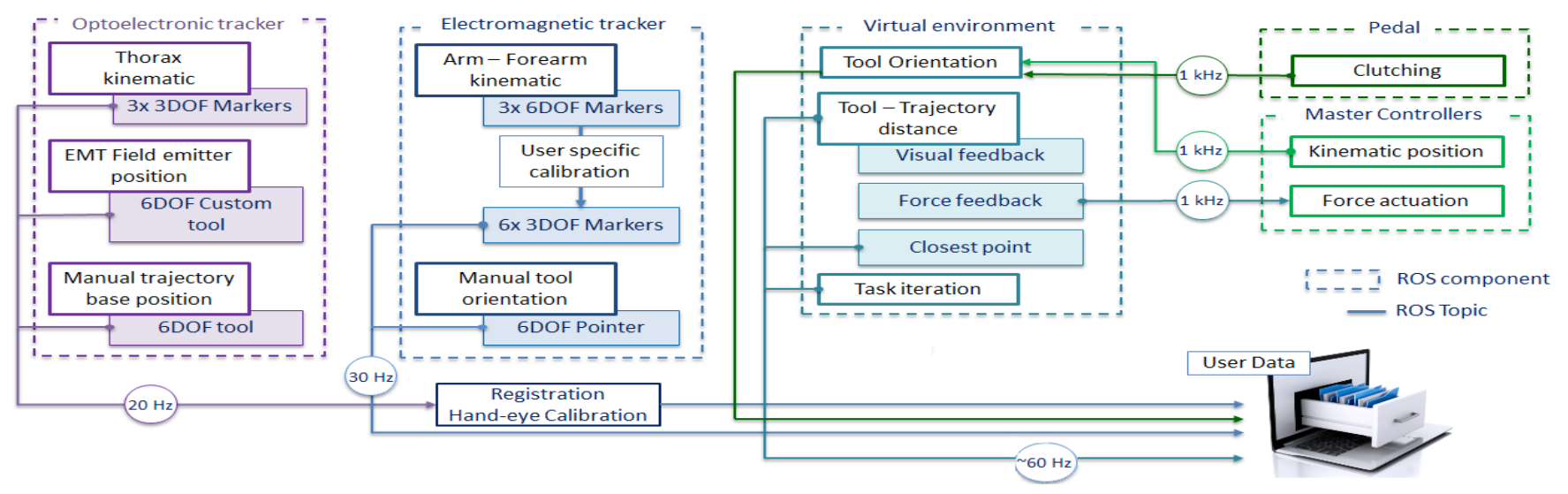

Fig. 2. The acquisition framework: each hatched box represents a ROS component that is responsible of streaming or monitoring a specific kind of signal. The arrows depict the ROS topic connecting each component and the acquisition frequencies are reported into the overlying circles

\section{Master devices}

To control the tool position and orientation in the virtual reality, two haptic devices were used. In both cases the use of a clutch was adopted to enlarge the robots' workspace. For both master devices a scaling factor of 0.5 was applied to the hand movement for the reconstruction of the tool position in the virtual scenarios. The Phantom Omni (3D Systems, South Carolina, USA) haptic device (Fig. 1(c))(workspace 160x120x70mm) was used as SL. The device is characterized by six dof with a stylus end-effector. The device is not gravity compensated and has a resolution of $0.055 \mathrm{~mm}$. An external pedal was used as clutch. As a controller, we used a custom developed impedance controller [25] based on the proprietary API. For PL, a gravity compensated Force Dimension Sigma.7 (Force Dimension, Nyon, Switzerland) haptic device (Fig. 1(d)) (workspace $\oslash 190 \times 130 \mathrm{~mm}$ ) was used. The robot is characterized by $6+1$ DoF delta based parallel kinematics with hand-centered rotation, it has a resolution of $0.0015 \mathrm{~mm}$ and $0.013 \mathrm{deg}$. An external pedal was used as a clutch. The device used the proprietary impedance controller.

\section{Acquisition architecture}

A custom data acquisition framework was developed to acquire, synchronize and record the different signals. Fig. 2 shows the ROS based acquisition framework, which allowed to assign a network timestamp at each sampled signal.

The component functionalities were monitored through a simple graphic interface, and they were running on three different computers, all based on Ubuntu 14.

The position of the arm was measured using an electromagnetic tracker (EMT) (Aurora - Northern Digital, Ontario, Canada, $30 \mathrm{~Hz}$ samplig rate, $0.48 \mathrm{~mm}$ and $0.3 \mathrm{deg}$ position and orientation RMSE, $500 \mathrm{~mm}$ dome shaped radius workspace). The system tracks four 6 DoF $1.8 \times 9 \mathrm{~mm}$ sensors.

Due to its limitation in both the maximal number of electromagnetic markers and the field dimension, the EMT system was coupled with an optical localization system (OPT) (Vicra - Northern Digital, Ontario, Canada, $20 \mathrm{~Hz}$ sampling rate, $0.25 \mathrm{~mm}$ position RMSE) that was used to acquire the thorax position. Three passive retroreflective markers were attached to the right and left acromions and beside the jugular notch. In order to register the data from the two tracking devices (EMT and OPT) an hand-eye calibration [26] was used. In order to perform the registration, a OPT tool $(R E F)$ was attached to the EMT field emitter. The homogeneous matrix that were computed, $R E F \mathbf{T}_{E M T}$, maps the transformation that brings the EMT reference frame on $R E F$ reference frame (see Fig. 3).

In the teleoperated tasks (PL and SL), the distance between the tool center and the closest point on the trajectory was computed in the virtual environment. In order to obtain this metric also in FH, an OPT tool (Base) was fixed to the wooden base at which the metal trajectory is rigidly connected (see Fig. 1(b)-1). 50 Points on the trajectory were acquired using the OPT pointer, and these coordinates were interpolated with a second order polynomial. The tool ring centre was recorded using an EMT pointer (see Fig. 1(b)-3) directly handled by the user terminating with a 3D printed ring tool (see Fig. 1(b)-4).

\section{E. The musculoskeletal model}

The subject musculoskeletal model was implemented in OpenSim [27]. The model derives from a 15 DoF model from Holzbaur [28] whose hand grip and fingers kinematic were fixed, simplifying it into a $7 \mathrm{DoF}$ model. Its performance in the dynamic prediction were previously demonstrated. [29]. The model is characterised by 50 Hill-type muscle-tendon actuators, simulating 32 muscle compartments. To fit the data from the different users that participated in the research, the model was scaled accordingly with the anthropometric characteristics, also adapting the different segments mass proprieties.

In order to be able to integrate the acquired markers data with the software, during patient calibration, we created virtual markers from the the electromagnetic sensors connected to the arm, forearm and hand segments (see Fig. 3).

Equation 1 describes the homogeneous transformation matrices that were used during the acquisition.

$$
{ }^{\text {Real }} \mathbf{T}_{\text {Virt }}=\left({ }^{E M T} \mathbf{T}_{\text {Real }}\right)^{-1} \cdot{ }^{E M T} \mathbf{T}_{\text {Virt }}
$$




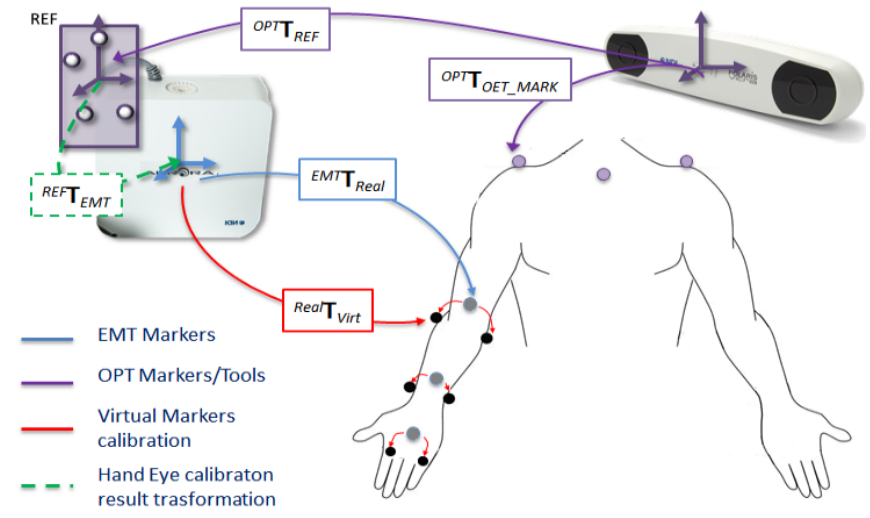

Fig. 3. Markers setup: gray points represent EMT markers position, i.e. arm, forearm and hand; black points are the virtual markers created during the calibration phase during which the EMT pointer tool was used to touch two recognisable bony landmarks on each arm segment, while a custom ROS component acquired the transformation that mapped the touched point in the EMT marker reference frame of the corresponding segment $\left({ }^{R e a l} \mathbf{T}_{\text {Virt }}\right)$. Arm and forearm sensors were connected to lateral and medial repere point of elbow and wrist respectively; the hand virtual sensors were placed on the index and little finger's knuckle.

$$
{ }^{E M T} \mathbf{T}_{\text {Virt }}={ }^{E M T} \mathbf{T}_{\text {Real }} \cdot{ }^{\text {Real }} \mathbf{T}_{\text {Virt }}
$$

where ${ }^{\text {Real }} \mathbf{T}_{\text {Virt }}$ is the transformation matrix that maps the virtual markers in the real markers reference frame, ${ }^{E M T} \mathbf{T}_{\text {Real }}$ is the transformation matrix that maps the real markers in the EMT reference frame and ${ }^{E M T} \mathbf{T}_{V i r t}$ is the transformation matrix that maps the virtual markers in the EMT reference frame.

The inverse kinematic reconstruction was carried on using the markers trajectories filtered with a second order low pass Butterworth filter at $4 \mathrm{~Hz}$. The inverse dynamic reconstruction was performed with the joint kinematic data from the inverse kinematic solution, filtered with the same $4 \mathrm{~Hz}$ low pass filter. The simulations were performed on an i5 4670k processor, operating at $3.8 \mathrm{Ghz}$ with $16 \mathrm{~Gb}$ of DDR3 memory.

\section{F. Stiffness computation}

Based on the analysis performed with OpenSim, the joint torque matrix $\mathbf{K j}$ for each arm configuration during the task execution was computed. The $(k \times k)$ joint stiffness matrix for the $i^{t h}$ sample of the acquisition can be defined as follows [30]:

$$
\mathbf{K} \mathbf{j}_{i}=\frac{d \boldsymbol{\tau}_{i}}{d \mathbf{q}_{i}}
$$

$\boldsymbol{\tau}_{i}$ is the ( $\left.k \times 1\right)$ vector that represents the joint torques for the $\mathbf{q}_{i}$ vector of joint angles and $k$ is the model's number of joints.

The joint torques vector is defined as:

$$
\tau_{i}=\mathbf{J m}_{i}^{T} \cdot \mathbf{F m}_{i}
$$

$\mathbf{F m}_{i}$ is the ( $\left.m \times 1\right)$ vector of the muscle activations (with $m$ number of muscular units in the model) at the $i^{t h}$ sample, obtained from the inverse dynamic analysis in OpenSim and $\mathbf{J m}_{i}$ is the muscle Jacobian at the $i^{t h}$ sample, computed as follows:

$$
\mathbf{J m}_{i}=\left(\begin{array}{cccc}
\frac{\partial l_{1, i}}{\partial q_{1, i}} & \frac{\partial l_{1, i}}{\partial q_{2, i}} & \cdot & \frac{\partial l_{1, i}}{\partial q_{k, i}} \\
\frac{\partial l_{2, i}}{\partial q_{1, i}} & \frac{\partial l_{2, i}}{\partial q_{2, i}} & \cdot & \frac{\partial l_{2, i}}{\partial q_{k, i}} \\
\cdot & \cdot & \cdot & \cdot \\
\frac{\partial l_{m, i}}{\partial q_{1, i}} & \frac{\partial l_{2, i}}{\partial q_{2, i}} & \cdot & \frac{\partial l_{m, i}}{\partial q_{k, i}}
\end{array}\right)
$$

The muscular Jacobian matrix, $\mathbf{J m}_{i}$, relates the variations in muscle length $\mathbf{l}_{i}$ with the variations in joint angular position $\mathbf{q}_{i}$ and can also be seen as the muscle forces moment arm matrix.

In order to study the stiffness characteristics and to create a graphical representation in the Cartesian space, the joint stiffness matrix at the $i^{\text {th }}$ sample was converted into the end-effector stiffness matrix $\mathbf{K e}_{i}$ using the corresponding Jacobian matrix $\mathbf{J}_{i}$ that relates infinitesimal changes in the Cartesian space into infinitesimal changes in the joint space.

$$
\mathbf{K} \mathbf{e}_{i}=\mathbf{J}_{i}^{-T} \mathbf{K} \mathbf{j}_{i} \mathbf{J}_{i}^{-1}
$$

From the singular value decomposition (SVD) of $\mathbf{K e}_{i}$ the translational stiffness property of the arm can be obtained at each arm configuration. The matrix $\mathbf{K e}_{i}$ has three nonzero singular values that define the ellipsoids main axes dimension while the ellipsoid orientation can be obtained from the $(3 \times 3)$ left singular vectors [30].

\section{G. Results analysis}

1) Error: As a measure of the task execution performance, in PL and SL the distance $(E)$ between the tool centre at the $i^{\text {th }}$ sample $\left(\mathbf{C}_{i}\right)$ and the closest point on the trajectory $(\mathbf{C P})$ was computed with a sampling frequency of $60 \mathrm{~Hz}(7)$

$$
E_{P L, i}=E_{S L, i}=\left\|\mathbf{C}_{i}-\mathbf{C P}_{i}\right\|
$$

where the norm indicates the Euclidean distance. In FH, through the registration procedure described in Section II$\mathrm{D}$, the tool center position was computed with respect to the trajectory. In order to obtain the coordinate of the closest point on the free hand trajectory $\left(\mathbf{C P F H}_{i}\right)$, the Quickhull algorithm [31] was used to find the closest point between the interpolated curve and each tool position $\mathbf{C}_{i}$ (see (8)).

$$
E_{F H, i}=\left\|\mathbf{C}_{i}-\mathbf{C P F H}_{i}\right\|
$$

The data distribution normality was tested using the Lilliefors test with a significance level $(\alpha)$ of 0.01 . Since no significant difference was found in the error distribution among users (Kruskal-Wallis H test $\alpha=0.001$ ), we grouped all the trials and users for each control modality into a single population. The differences between the three control modalities were evaluated through a post-hoc multivariate analysis using the Willcoxon rank sum test $(\alpha=0.001)$.

2) Stiffness ellipsoids - Main axis length: Fig. 4(a) represents the stiffness ellipsoid obtained in FH task execution. The ellipsoid main axis length $\left(K_{\max }\right)$ was computed for six points $(P 1 \div P 6)$ along the trajectory (see Fig. 4(b))

The population normality was tested using the Lilliefors test $(\alpha=0.01)$. Since no statistical difference was found between different users and repetitions (Kruskal-Wallis $\mathrm{H}$ 


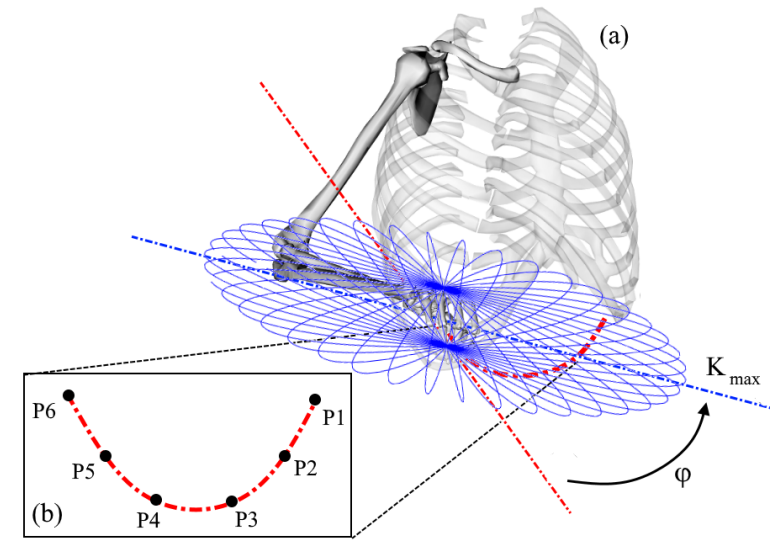

Fig. 4. The computed ellipsoid (in blue) is overlapped to the muscoloskeletal model at a specific point on the trajectory (in red). (a) The main axis dimension $K_{\max }$ and its angle with the tangent to the trajectory $\varphi$ are monitored through the different users task repetitions. (b) The six points along the trajectory

test $\alpha=0.001$ ), the $K_{\max }$ values for each point through the users and repetitions were grouped together. We grouped the data from the six point along the trajectory together, evaluating the statistical differences between the three control modalities using multiple Willcoxon rank sum tests $(\alpha=$ $0.001)$.

3) Stiffness ellipsoids - Angular displacement: The angle between the main axis and the tangent to the trajectory $(\varphi)$ was computed for the same six points $(P 1 \div P 6)$. The value was computed obtaining the vector tangent to the desired trajectory in the six points and the vector representing the instantaneous end-effector direction of motion. Since no statistical difference was found between different users and repetitions (Kruskal-Wallis $\mathrm{H}$ test $\alpha=0.001$ ), the angular displacement $\varphi$ values for each point through the users and repetitions were grouped together. The existence of possible parabolic trends in the angular displacement through the trajectory was evaluated using a second order polynomial regression.

\section{RESULTS}

\section{A. Error}

Fig. 5 shows the error data distribution in the three control modalities (PL, SL and FH). PL median error is statistically lower that SL and FH $(p<0.001)$. In general, teleoperation (PL and SL) allowed the users to execute the task with higher accuracy with respect to free-hand.

\section{B. Stiffness Ellipsoids - Main axis length}

Fig. 6 shows the distribution of the maximal end-point stiffness in the six trajectory points and the three controlling modalities. The results obtained show that, during tasks execution, a parabolic trend could be seen in all the control modalities: while for SL and FH the maximal values seems to be occurring for the central point of the trajectory (P3 and $\mathrm{P} 4)$, this trend is opposite in PL, where at the central points of the trajectory correspond the smallest values of maximal stiffness.

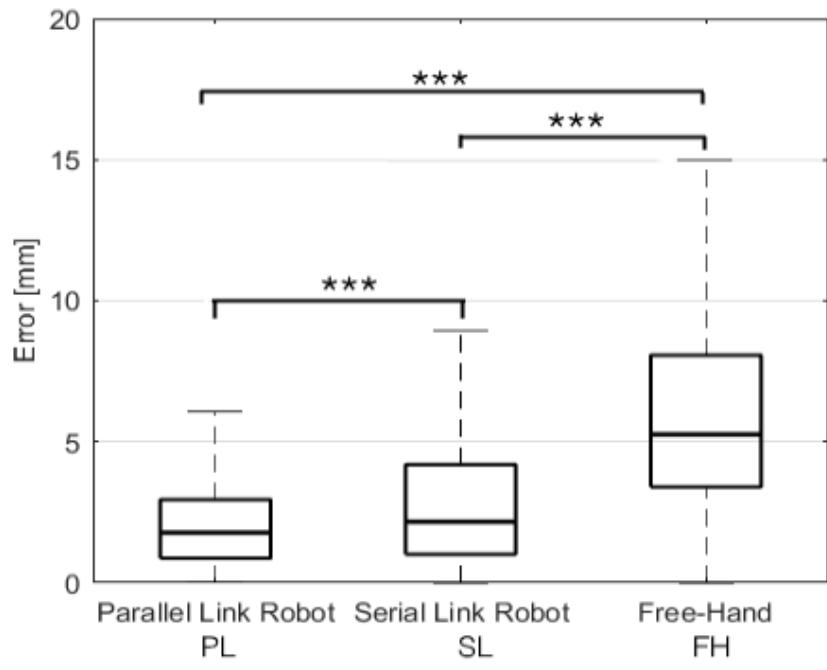

Fig. 5. The error metric in PL, SL, and FH are reported as $25^{t h}$ and $75^{t h}$ percentiles and median values. The statistical difference between the control modalities is reported using horizontal lines. Three stars indicate $p<0.001$

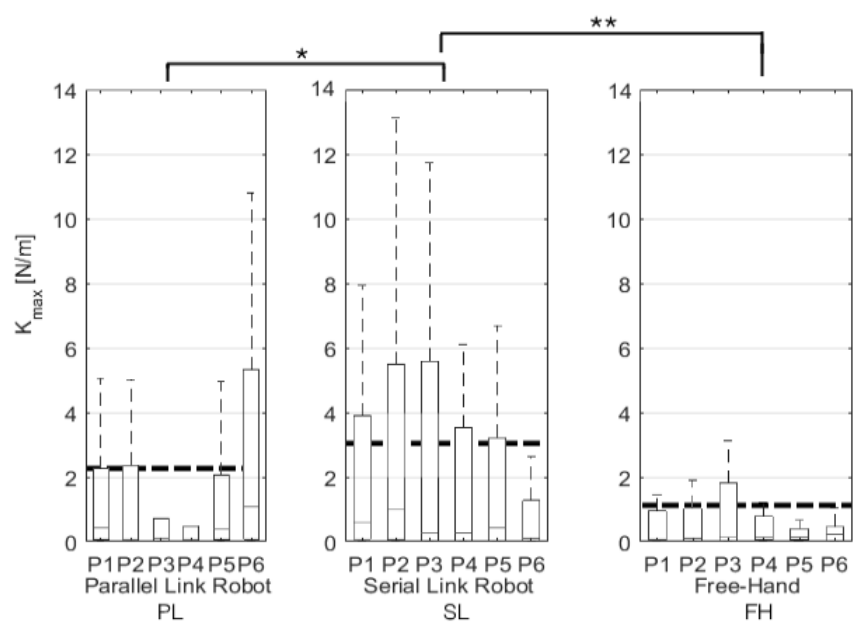

Fig. 6. The maximal hand stiffness in the six points of the trajectory for the parallel link robot, for the serial link robot and for free-hand. Boxes indicate the $25^{t h}$ and $75^{t h}$ percentiles, vertical dashed lines indicate the maximal values and the horizontal dashed lines represent the median values of the three populations. The horizontal lines at the top of the graph describe the statistical difference between the control modalities when all the six points are considered together, as in Table I. One star correspond to $p<0.05$, two stars to $p<0.01$

Table I reports the stiffness maximal values $K_{\max }$ distributions for the six points grouped in the three control modalities. The median values are also reported as dashed lines in Fig. $6(\mathrm{PL}=0.21 \mathrm{~N} / \mathrm{m}, \mathrm{SL}=0.32 \mathrm{~N} / \mathrm{m}, \mathrm{FH}=0.12$ $\mathrm{N} / \mathrm{m})$.

The results show that SL maximal stiffness values are statistically higher from both PL (with a $p<0.05$ ) and FH $(p<0.01)$.

\section{Stiffness Ellipsoids - Angular displacement}

Fig. 7 shows the evolution of the angle $\varphi$ in the six points of the trajectory for the three control modalities. Analysing the angular displacement results, a parabolic trend can be found in the median values obtained through the six points 
TABLE I

MAXIMAL STIFFNESS $K_{\max }$ IN THE THREE CONTROL MODALITIES

\begin{tabular}{cccc}
\hline & $\begin{array}{c}\text { Parallel Link Robot } \\
\text { PL }\end{array}$ & $\begin{array}{c}\text { Serial Link Robot } \\
\text { SL }\end{array}$ & $\begin{array}{c}\text { Free-hand } \\
\text { FH }\end{array}$ \\
\hline Min: & $0.00 \mathrm{~N} / \mathrm{m}$ & $0.00 \mathrm{~N} / \mathrm{m}$ & $0.00 \mathrm{~N} / \mathrm{m}$ \\
$25^{t h}$ p.: & $0.04 \mathrm{~N} / \mathrm{m}$ & $0.04 \mathrm{~N} / \mathrm{m}$ & $0.04 \mathrm{~N} / \mathrm{m}$ \\
Median: & $0.21 \mathrm{~N} / \mathrm{m}$ & $0.32 \mathrm{~N} / \mathrm{m}$ & $0.12 \mathrm{~N} / \mathrm{m}$ \\
$75^{t h}$ p.: & $3.60 \mathrm{~N} / \mathrm{m}$ & $4.00 \mathrm{~N} / \mathrm{m}$ & $0.71 \mathrm{~N} / \mathrm{m}$ \\
Max: & $5.55 \mathrm{~N} / \mathrm{m}$ & $8.73 \mathrm{~N} / \mathrm{m}$ & $1.45 \mathrm{~N} / \mathrm{m}$ \\
\hline
\end{tabular}

on the trajectory. The R-square indexes obtained for PL, SL and FH are respectively $0.32,0.43$ and 0.66 (Fig. 7 - dotted lines).

This behaviour, which is particularly clear in the freehand control, tends to align the stiffness main axis in order to make it perpendicular to the desired direction of motion in correspondence to $\mathrm{P} 3$ and $\mathrm{P} 4$, the central points of the trajectory.
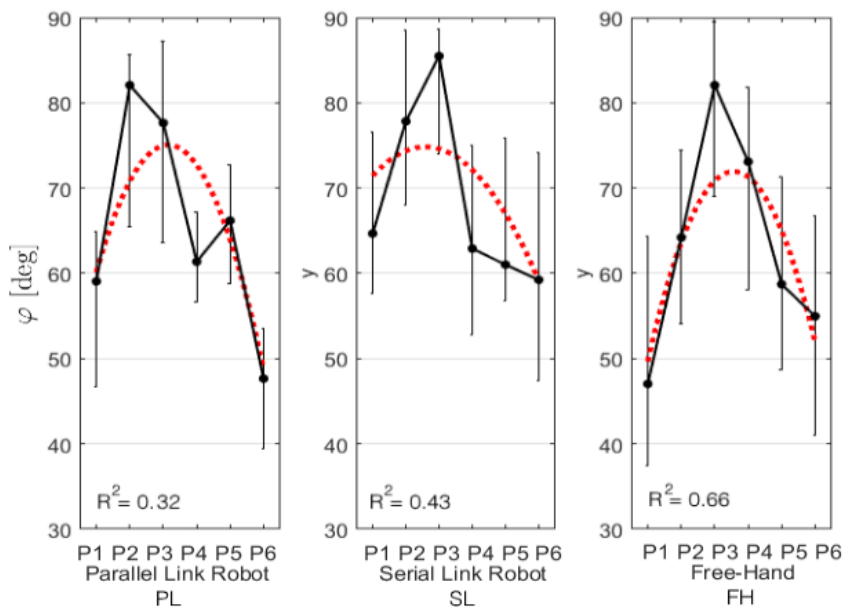

Fig. 7. The angle between the maximal hand stiffness direction and the tangent to the desired trajectory in the six points for the three control modalities. The points represent the median values, while the vertical lines represent the $25^{t} h$ and $75^{t} h$ percentiles. The dotted line represents the second order polynomial regression, with the corresponding $R^{2}$ values associated.

\section{Discussion}

In this work we studied the arm end-point stiffness as a measure of the impedance control strategies adopted by the central nervous system to stabilize the hand movement in three tasks, in teleoperation, executed with two master devices, and in free-hand. A task mimicking suturing was realised in a virtual and real environment;

As expected, we found that the error metric results reflect the strength of the teleoperation scenario in reducing the influence of the hand control variability: the tasks error are smaller in teleoperation with respect to free-hand thanks to the motion scaling and the color feedback that was used to finely adjust the tool position.

By analysing the results obtained in the $K_{\max }$ modulation, there are significant differences between the control modalities. In SL control modality only, the users were exploiting the pivoting stylus to execute the trajectory. In PL, instead, the rotations were hand centred, as in $\mathrm{FH}$, where the dimensions of the tool and its weight discouraged the users from executing the task mainly activating the wrist joints. It is possible that the presence of this "lever" in SL forced the users to increase the arm stiffness to achieve good performance. This result suggests that hand-centred rotations in the manipulator allow the users to achieve statistically higher kinematic performances while eliciting lower arm stiffness. Since lower arm stiffness values are obtained with lower levels of muscular activation, hand-centred rotating master devices could reduce the users' muscular fatigue and should therefore be preferred.

Regarding $\varphi$ modulation, in FH the regression curve obtained is partially able to fit the data, suggesting the possible existence of a parabolic behaviour. This behaviour could be the result of a motor strategy that aims at maximising the hand resistance to external perturbation that would directly influence the task execution while maintaining minimal stiffness in the direction of motion. The maximal values of $\varphi$ are achieved in P3 and P4, where the users had to change their wrist configuration to follow the trajectory curvature; from this result it seems that the users were relying on the directional control of stiffness in particular in the most challenging part of the trajectory. If this evidence would be confirmed by more in depth researches, it would be possible to actively use the master devices' force feedback to increase the stiffness on the directions perpendicular to the motion, reducing the total muscular activation requested to the arm. Future works will also test the hypothesis of stiffness regulation with respect to motion direction in order to understand how the master devices design could influence the possibility of putting this motor strategy into practice.

The first limit of this work can be found in the high variability of the data related to the stiffness ellipsoids. Both the maximal stiffness $K_{\max }$ and the angular displacement $\varphi$ suffer from high interquartile dispersion inter and intra users. A reason for this behaviour could be found in the complexity of the tasks, that represents a difficult precision task, and presents different challenges for each user. In spite of the task being simplified from a previous set of acquisition, this variability, even though reduced, still persist. An other limitation can be found in the joint stiffness computation, that does not take into consideration possible voluntary muscle co-contractions.

Even though it presents some limitations, this study represent a novel approach to the study of the human robot interaction in teleoperation. The results obtained could be used as an optimization goal for the design of new master devices.

\section{FUtURE DEVELOPMENTS}

Starting from this results, it would be desirable to extend the analysis to more subjects and more trajectories, creating task on different planes to test the users capability in modulating the hand stiffness orientation. 
An interesting future development will be the comparison between the stiffness computation method presented in this work and other methods: for example, during the tasks execution, known external forces could be applied, acting as random perturbations on the users hand; the measured displacement induced in the hand position could be used to directly compute the hand stiffness. Another possible comparison could be done with the methods that use electromyography signals to evaluate the arm stiffness. It would also be interesting to acquire the electromyographic activation of the muscles that control the upper limb movements, performing a co-contraction analysis aimed at finding a physiological correspondence to the analytical stiffness modulation that was found.

\section{ACKNOWLEDGEMENTS}

This project has received funding from the European Union's Horizon 2020 research and innovation programme under grant agreement No. H2020-ICT-2016-732515.

\section{REFERENCES}

[1] M. Talamini, S. Chapman, S. Horgan, and W. S. Melvin, "A prospective analysis of 211 robotic-assisted surgical procedures," Surgical Endoscopy and Other Interventional Techniques, vol. 17, no. 10, pp. 1521-1524, 2003.

[2] A. R. Lanfranco, A. E. Castellanos, J. P. Desai, and W. C. Meyers, "Robotic surgery: a current perspective," Annals of surgery, vol. 239, no. 1, pp. 14-21, 2004.

[3] R. McVey, M. G. Goldenberg, M. Q. Bernardini, K. Yasufuku, F. A. Quereshy, A. Finelli, K. T. Pace, and J. Y. Lee, "Baseline laparoscopic skill may predict baseline robotic skill and early robotic surgery learning curve," Journal of Endourology, vol. 30, no. 5, pp. 588-592, 2016.

[4] S. Kaul, N. L. Shah, and M. Menon, "Learning curve using robotic surgery," Current urology reports, vol. 7, no. 2, pp. 125-129, 2006.

[5] J. Hernandez, S. Bann, Y. Munz, K. Moorthy, V. Datta, S. Martin, A. Dosis, F. Bello, A. Darzi, and T. Rockall, "Qualitative and quantitative analysis of the learning curve of a simulated surgical task on the da vinci system," Surgical Endoscopy And Other Interventional Techniques, vol. 18, no. 3, pp. 372-378, 2004.

[6] G. H. Ballantyne and F. Moll, "The da vinci telerobotic surgical system: the virtual operative field and telepresence surgery," Surgical Clinics of North America, vol. 83, no. 6, pp. 1293-1304, 2003.

[7] N. Enayati, E. De Momi, and G. Ferrigno, "Haptics in robot-assisted surgery: challenges and benefits," IEEE reviews in biomedical engineering, vol. 9, pp. 49-65, 2016.

[8] E. Beretta, F. Nessi, G. Ferrigno, and E. De Momi, "Force feedback enhancement for soft tissue interaction tasks in cooperative robotic surgery," in Intelligent Robots and Systems (IROS), 2015 IEEE/RSJ International Conference on. IEEE, 2015, pp. 209-215.

[9] T. Ortmaier, B. Deml, B. Kübler, G. Passig, D. Reintsema, and U. Seibold, "Robot assisted force feedback surgery," in Advances in telerobotics. Springer, 2007, pp. 361-379.

[10] N. Enayati, E. C. A. Costa, G. Ferrigno, and E. De Momi, "A dynamic non-energy-storing guidance constraint with motion redirection for robot-assisted surgery," in Intelligent Robots and Systems (IROS), 2016 IEEE/RSJ International Conference on. IEEE, 2016, pp. 4311-4316.

[11] E. Beretta, F. Nessi, G. Ferrigno, F. D. Meco, A. Perin, L. Bello, G. Casaceli, F. Raneri, A. D. Benedictis, and E. D. Momi, "Enhanced torque-based impedance control to assist brain targeting during openskull neurosurgery: a feasibility study," The International Journal of Medical Robotics and Computer Assisted Surgery, vol. 12, no. 3, pp. 326-341, jul 2015.

[12] E. Beretta, E. De Momi, F. R. y Baena, and G. Ferrigno, "Adaptive hands-on control for reaching and targeting tasks in surgery," International Journal of Advanced Robotic Systems, vol. 12, no. 5, p. 50, 2015.
[13] R. Sigrist, G. Rauter, R. Riener, and P. Wolf, "Augmented visual, auditory, haptic, and multimodal feedback in motor learning: a review," Psychonomic bulletin \& review, vol. 20, no. 1, pp. 21-53, 2013.

[14] I. Nisky, A. M. Okamura, and M. H. Hsieh, "Effects of robotic manipulators on movements of novices and surgeons," Surgical endoscopy, vol. 28, no. 7, pp. 2145-2158, 2014.

[15] I. Nisky, M. H. Hsieh, and A. M. Okamura, "The effect of a robotassisted surgical system on the kinematics of user movements," in 2013 35th Annual International Conference of the IEEE Engineering in Medicine and Biology Society (EMBC). IEEE, 2013, pp. 62576260.

[16] F. A. Mussa-Ivaldi, N. Hogan, and E. Bizzi, "Neural, mechanical, and geometric factors subserving arm posture in humans," The Journal of neuroscience, vol. 5, no. 10, pp. 2732-2743, 1985.

[17] E. Burdet, R. Osu, D. Franklin, T. Yoshioka, T. Milner, and M. Kawato, "A method for measuring endpoint stiffness during multi-joint arm movements," Journal of biomechanics, vol. 33, no. 12, pp. 1705-1709, 2000.

[18] P. L. Gribble, L. I. Mullin, N. Cothros, and A. Mattar, "Role of cocontraction in arm movement accuracy," Journal of neurophysiology, vol. 89, no. 5, pp. 2396-2405, 2003.

[19] B. Laursen, B. R. Jensen, and G. Sjøgaard, "Effect of speed and precision demands on human shoulder muscle electromyography during a repetitive task," European Journal of Applied Physiology and Occupational Physiology, vol. 78, no. 6, pp. 544-548, 1998.

[20] D. W. Franklin, R. Osu, E. Burdet, M. Kawato, and T. E. Milner, "Adaptation to stable and unstable dynamics achieved by combined impedance control and inverse dynamics model," Journal of neurophysiology, vol. 90, no. 5, pp. 3270-3282, 2003.

[21] M. Darainy, N. Malfait, P. L. Gribble, F. Towhidkhah, and D. J. Ostry, "Learning to control arm stiffness under static conditions," Journal of neurophysiology, vol. 92, no. 6, pp. 3344-3350, 2004.

[22] H. Gomi and M. Kawato, "Human arm stiffness and equilibriumpoint trajectory during multi-joint movement," Biological cybernetics, vol. 76, no. 3, pp. 163-171, 1997.

[23] D. Shin, J. Kim, and Y. Koike, "A myokinetic arm model for estimating joint torque and stiffness from emg signals during maintained posture," Journal of neurophysiology, vol. 101, no. 1, pp. 387-401, 2009.

[24] M. Quigley, K. Conley, B. Gerkey, J. Faust, T. Foote, J. Leibs, R. Wheeler, and A. Y. Ng, "Ros: an open-source robot operating system," in ICRA workshop on open source software, vol. 3, no. 3.2. Kobe, 2009 , p. 5.

[25] N. Hogan, "Impedance control: An approach to manipulation," in American Control Conference, 1984. IEEE, 1984, pp. 304-313.

[26] R. Horaud and F. Dornaika, "Hand-eye calibration," The international journal of robotics research, vol. 14, no. 3, pp. 195-210, 1995.

[27] S. L. Delp, F. C. Anderson, A. S. Arnold, P. Loan, A. Habib, C. T. John, E. Guendelman, and D. G. Thelen, "Opensim: open-source software to create and analyze dynamic simulations of movement," IEEE transactions on biomedical engineering, vol. 54, no. 11, pp. 1940-1950, 2007.

[28] K. R. Holzbaur, W. M. Murray, and S. L. Delp, "A model of the upper extremity for simulating musculoskeletal surgery and analyzing neuromuscular control," Annals of biomedical engineering, vol. 33, no. 6, pp. 829-840, 2005.

[29] K. R. Saul, X. Hu, C. M. Goehler, M. E. Vidt, M. Daly, A. Velisar, and W. M. Murray, "Benchmarking of dynamic simulation predictions in two software platforms using an upper limb musculoskeletal model," Computer methods in biomechanics and biomedical engineering, vol. 18, no. 13, pp. 1445-1458, 2015.

[30] J. McIntyre, F. Mussa-Ivaldi, and E. Bizzi, "The control of stable postures in the multijoint arm," Experimental brain research, vol. 110, no. 2, pp. 248-264, 1996.

[31] C. B. Barber, D. P. Dobkin, and H. Huhdanpaa, "The quickhull algorithm for convex hulls," ACM Transactions on Mathematical Software (TOMS), vol. 22, no. 4, pp. 469-483, 1996. 